\title{
Ernährungsumstellung, Supplementation und Bewegungsprogramm nach Roux-Y-Magenbypass
}

\author{
Kaja Ludwig Jörn Bernhardt Sylke Schneider-Koriath Uwe Scharlau Reiko Wießner
}

Klinik für Chirurgie, Klinikum Südstadt Rostock, Deutschland

\section{Schlüsselwörter \\ Morbide Adipositas · Roux-Y-Magenbypass · Supplementa- tion · Mangelzustände · Bewegungsprogramm}

\section{Zusammenfassung}

Die Zahl der notwendigen Magenbypass-Operationen (RYGB) steigt aufgrund der Adipositasepidemie jährlich an. Dabei wird ein Erfolg dieser Behandlung nur garantiert, wenn im Nachsorgeprogramm eine entsprechende Bewegungstherapie, eine verfahrensadaptierte Ernährungsumstellung und eine ausreichende Supplementation greifen. In Abhängigkeit von muskuloskelettalen Vorerkrankungen sollte eine Bewegungstherapie circa 4 Wochen postoperativ beginnen und aus einem abwechselnd kardiovaskulären und bindegewebsrestitutiven Training bestehen. Die erforderliche Ernährungsumstellung fokussiert auf eine klein portionierte, kalorienreduzierte und eiweiß- bzw. vitaminangereicherte Ernährung. Das tägliche Richtmaß sollte zwischen 800 und 1200 kcal liegen. Trotzdem können nach RYGB nutritive Mangelzustände für Proteine in 1-3\%, für Eisen in $45-52 \%$, Vitamin $B_{12}$ in 33$37 \%$, Folsäure in circa $35 \%$, Calcium in $10-12 \%$ und für Vitamine in $10-45 \%$ der Fälle registriert werden. Aus diesem Grund ist eine regelmäßige Laboranalytik im Follow-up notwendig, und es muss eine entsprechende Supplementation von Mineralien, Vitaminen und Spurenelementen durchgeführt werden.

\section{Einleitung}

In den letzten Jahren ist der Magenbypass (Roux-en-Y Gastric Bybass; RYGB) der weltweit häufigste Eingriff in der bariatrischen Chirurgie geworden [1-3]. Die Operation ist inzwischen laparosko-

\section{Key Words}

Morbid obesity · Roux-en-Y gastric bypass · Supplementation · Nutritional deficiencies · Physical activity

\section{Summary}

Nutritional Recommendations, Supplementation, and Physical Activity Program Following Roux-en-Y Gastric Bypass for Morbid Obesity

The number of gastric bypass operations (RYGB) needed worldwide is increasing annually due to the obesity epidemic. Yet the success of this treatment is only guaranteed if an appropriate exercise therapy, a corresponding change of diet, and an adequate supplementation take hold in the aftercare program. Subject to pre-existing musculoskeletal diseases, exercise therapy should start about 4 weeks after the operation and comprise alternating cardiovascular and connective tissue-restitution training. The required change of diet focuses on small portions of calorie-reduced as well as protein- and vitaminenriched food. The standard daily intake should be between 800 and 1,200 kcal. However, after RYGB, nutritive deficiencies have been registered for proteins in $1-3 \%$, for iron in $45-52 \%$, vitamin $\mathrm{B}_{12}$ in $33-37 \%$, folic acid in about $35 \%$, calcium in 10 $12 \%$, and vitamins in $10-45 \%$ of the patients. For this reason, laboratory analysis at regular intervals is necessary in the follow-up and an appropriate supplementation of minerals, vitamins, and trace elements must be implemented.

pisch sicher durchführbar und weist ein perioperatives Letalitätsrisiko von lediglich $0,2-2 \%$ auf $[4,5]$. In Studien mit einem Langzeit-Follow-up von bis zu 15 Jahren wird ein durchschnittlicher Excess Weight Loss (EWL) von 52-85\% nach RYGB angegeben $[6,7]$.

\section{KARGER \\ (C) 2009 S. Karger GmbH, Freiburg \\ Fax +497614520714 \\ E-mail Information@Karger.de www.karger.com/ofa \\ www.karger.com}

Prof. Dr. Kaja Ludwig

Klinik für Chirurgie, Klinikum Südstadt Rostock

Südring 81, 18059 Rostock, Germany

Tel. +49 38144 01-40 00, Fax -40 99

kfch@kliniksued-rostock.de 
Für den Erfolg eines RYGB sind eine geeignete Patientenselektion und insbesondere eine strukturierte und kompetente Nachsorge verantwortlich. Im Zentrum dieser Nachsorge stehen zum einen die Erkennung und Behandlung möglicher postoperativer Komplikationen sowie der Adipositas-assoziierten Komorbidität. Zum anderen muss potentiellen Mangelerscheinungen im Zusammenhang mit der malabsorptiven Komponente des Verfahrens durch präventive und/oder therapeutische Supplementation begegnet werden. Letztlich stützt neben der erforderlichen Ernährungsumstellung auch ein systematisches Bewegungsprogramm die erfolgreiche Gewichtsabnahme, die kardiovaskuläre Restitution und die Bindegewebsrückbildung der morbid adipösen Patienten $[8,9]$.

\section{Diätempfehlungen nach RYGB}

Wenn keine operativen Besonderheiten beachtet werden müssen, kann ein stufenweiser Kostaufbau zügig nach der Operation begonnen werden. Dabei wird den Patienten bis zum stationären Entlassungszeitpunkt die Aufnahme von flüssigen bzw. breiigen Speisen erlaubt. Erst nach 4 Wochen ist der Wechsel auf feste Nahrung zu empfehlen, wobei anfänglich leicht verdauliche Nahrungskomponenten (z. B. pürierte Kost, kurzfaseriges Fleisch) zu bevorzugen sind. Später können je nach Verträglichkeit auch alle übrigen kalorienarmen Nahrungsmittel in den täglichen Kost- und Ernährungsplan integriert werden. Obligat ist die Führung eines Ernährungsprotokolls. Ernährungshinweise nach bariatrischen Operationen sollten sowohl diätetischen als auch verfahrenstechnischen Gesichtspunkten nach RYGB entsprechen und gleichzeitig Mangelerscheinungen vorbeugen. Dabei haben sich gewisse Grundregeln etabliert, welche allgemein Anwendung finden können (Tab. 1). Empfehlenswert ist die Umstellung des Essverhaltens auf nicht mehr als 3-4 Mahlzeiten pro Tag mit einer Gesamtenergieaufnahme von $800-1200 \mathrm{kcal} /$ Tag. Zwischenmahlzeiten sollten vermieden werden, denn sie stimulieren die Insulinausschüttung und hemmen somit den Fettabbau. Durch das kleine Magenreservoir nach RYGB (10-20 ml) können nur begrenzte Nahrungsmengen aufgenommen werden. Aus diesem Grund sollten Essen und Trinken getrennt werden. Um eine ausreichende Flüssigkeitsaufnahme zu gewährleisten, ist das regelmäßige Trinken (150-300 ml/h) zwischen den Mahlzeiten notwendig. Kohlensäurehaltige Getränke sind strikt zu vermeiden [10]. Alkoholgenuss sollte ebenfalls unterbleiben, nicht nur weil Alkohol neben Fett der größte Kalorienträger ist, sondern weil in vielen Fällen eine beschleunigte Resorption im Dünndarm erfolgt und die Gefahr einer alkoholinduzierten Leberzirrhose deutlich steigt.

Proteine stellen unsere wichtigste Nahrungsquelle dar. Deshalb ist nach RYGB folgende Essreihenfolge zu empfehlen: zuerst Proteine, dann Gemüse und Früchte und erst abschließend Kornprodukte respektive Kohlenhydrate. Klinisch relevante Proteinmangelzustände sind nach RYGB bei nicht mehr als 3-4\% der Patienten festzustellen [10]. Brolin und Leung [11] fanden in einer Umfrage unter 109 bariatrischen Chirurgen mit knapp 45000 malabsorptiven Eingriffen eine Rehospitalisierungsrate von lediglich 0,003\%
Tab. 1. Empfohlene Diätregeln nach RYGB [10]

\begin{tabular}{l}
\hline Ernährungsregeln \\
\hline 1. Langsam essen, häufig kauen \\
2. Essen und Trinken trennen \\
3. Ständig zwischen den Mahlzeiten trinken $(150-300 \mathrm{ml} / \mathrm{h})$ \\
4. Keine Süßigkeiten, kein Alkohol \\
5. Viele Vitamine essen \\
6. Täglich 3-4 Mahlzeiten, möglichst keine Zwischenmahlzeiten \\
7. Bei Sättigung aufhören zu essen
\end{tabular}

nach RYGB und 0,05\% nach biliopankreatischer Diversion (BPD), bei der eine total parenterale Ernährung wegen Hypalbuminämie notwendig wurde. Dagegen wird bei einer diätetisch fehlerhaften Zuckeraufnahme bei bis zu 76\% ein Dumping-Syndrom beschrieben, wobei durch eine entsprechende Operationstechnik und eine umsichtige Patienteninformation eine permanente Rate von $<16-20 \% \mathrm{zu}$ erreichen ist $[12,13]$.

Entscheidend für einen Erfolg nach RYGB ist neben der Ernährungsumstellung ein ausreichendes Wissen des Patienten über die Wirkungsweise der durchgeführten Operation und mögliche Symptome, die Komplikationen oder Mangelerscheinungen nach RYGB signalisieren.

\section{Supplementation nach RYGB}

In mehreren Studien konnte gezeigt werden, dass auch bei Beachtung aller Ernährungsregeln aufgrund der malabsorptiven Komponente des RYGB mit Mangelerscheinungen gerechnet werden muss (Tab. 2) [14-17].

Serum-Eisendefizite werden nach RYGB bei über der Hälfte der Patienten beschrieben, eine begleitende Anämie bei bis zu $74 \%$ $[11,16,18]$. Die Hauptursache dürfte zum einen in der Ausschaltung von Duodenum und oberem Jejunum sowie in der fehlenden präabsorptiven Umwandlung des Eisens durch die Magensäure liegen. Zum anderen werden Hauptnahrungsquellen für Eisen wegen Unverträglichkeit häufig gemieden. Damit ergibt sich die Notwendigkeit einer ausreichenden Substitution, insbesondere bei prämenopausalen Patientinnen, obgleich selbst bei Tagesdosen von bis zu $750 \mathrm{mg}$ eine komplette Normalisierung nicht erreicht werden kann.

Vitamin- $\mathrm{B}_{12}$-Defizite wurden bei bis zu $64 \%$ der Patienten registriert $[18,19]$. Auch hier scheiden die Hauptnahrungsquellen (z. B. Milch und rotes Fleisch) für viele Operierte aufgrund von Intoleranzen aus. Obgleich Vitamin $\mathrm{B}_{12}$ überwiegend im Ileum resorbiert wird, fehlt durch die Ausschaltung des Magens und damit des aufspaltenden Einflusses der Magensäure die Lösung des Vitamins aus der Nahrung. Zusätzlich weisen Untersuchungen darauf hin, dass die Sekretionsleistung für IF (intrinsic factor) im ausgeschalteten Magen reduziert ist. Aus diesem Grund kann eine großzügige Empfehlung zur Supplementation mit $500 \mu \mathrm{g} / \mathrm{Tag}$ oral gegeben werden. Effektiver scheint die subkutane Applikation (1000$2000 \mu \mathrm{g} / 1-3$ Monate) zu sein $[10,16]$. 
Tab. 2. Nährstoffdefizite nach RYGB (zum Teil Patienten nach RYGB mit unzureichender Supplementation eingeschlossen [10, 16, 18, 21-23])

\begin{tabular}{lcc}
\hline & \multicolumn{2}{c}{ Patienten mit Defiziten, \% } \\
\cline { 2 - 3 } & distaler RYGB & proximaler RYGB \\
\hline Protein & $3-13$ & $1,4-3$ \\
Eisen & $23-45$ & $45-52$ \\
Vitamin B & $8-33$ & $33-37$ \\
Folsäure & $32-63$ & 35 \\
Calcium & 25 & $10-12$ \\
Vitamin D & $51-79$ & $45-49$ \\
Fettlösliche Vitamine & $4-18$ & $10-23$ \\
\hline
\end{tabular}

Problematischer sind Mangelzustände im Bereich des Calciumund Vitamin-D-Stoffwechsels. Serumanalysen zeigen zwar ein Calciumdefizit nur bei circa $10-12 \%$ der Patienten nach RYGB und circa $25 \%$ nach BPD [20]. Allerdings sollte in diesem $\mathrm{Zu}$ sammenhang beachtet werden, dass im Rahmen einer SerumCalcium-Regulation bei Mangelzuständen zusätzliches Calcium aus dem Knochen mobilisiert wird und damit gleichzeitig einem möglichen sekundären Hyperparathyroidismus (sHPT) bzw. einer Osteoporose bei bis zu 25-40\% der Patienten Vorschub leistet. Johnson et al. [21] fanden bei 243 Patienten nach RYGB sogar in $88 \%$ der Fälle erhöhte PTH-Levels, wobei $42 \%$ dieser Patienten postoperativ normale Vitamin-D-Werte aufwiesen. Vorzugsweise sind in diesem Zusammenhang postmenopausale Frauen betroffen. Coates et al. [22] konnten unter diesen Voraussetzungen bei normocalcämischen Patienten eine erhöhte Calciumfreisetzung aus dem Knochen mittels erhöhter Marker (Osteocalcin und N-Telopeptid) nachweisen. Somit ist die sofortige Calcium-Substitution mit 1000-2000 mg/Tag unmittelbar postoperativ notwendig, obgleich in Studien festgestellt wurde, dass dadurch ein existenter sHPT nicht normalisiert werden konnte. Allerdings wiesen 25\% der obesen Patienten bereits vor der Operation einen prädispositionellen oder manifesten sHPT auf $[16,20,22]$.

Vitamin-D-Defizite können bei circa 50\% der Patienten auftreten. Die Ursache dürfte in einer verminderten Resorptionsleistung aufgrund einer ungenügenden Absorptionsvorbereitung des fettlöslichen Vitamins im Ileum liegen. DiGiorgi et al. analysierten $400 \mathrm{~Pa}-$ tienten vor und nach RYGB. Sie fanden in $50 \%$ der Fälle präoperative Mangelzustände, welche postoperativ zwar unter adäquater Supplementation eine Optimierung des Obesity-korrelierten negativen Knochenmetabolismus zeigten, jedoch auch erhöhte PTHSerumlevel und reduzierte Vitamin-D-Werte aufwiesen [23]. In der oben genannten Studie von Johnson et al. [21] fanden die Autoren in einer Subgruppenanalyse zwischen Patienten mit LongLimb- und Short-Limb-RYGB (alimentärer Schenkel >100 vs. $<100 \mathrm{~cm}$ ) signifikant häufiger einen Vitamin-D-Mangel nach Long-Limb-RYGB (79 vs. 49\%). Aus diesem Grund ist neben einer laboranalytischen Nachsorgeüberwachung die tägliche Substitution mit 400 IU anzuraten, wobei Patienten jenseits des 60. Lebensjahres einen etwas erhöhten Bedarf haben (700 IU/Tag).

Vitamin-A-Mangelzustände sind extrem selten klinisch relevant und können ohne Probleme durch eine modifizierte Nahrungszu-
Tab. 3. Möglicher Supplementationsbedarf nach RYGB

\begin{tabular}{|c|c|}
\hline & Empfohlene Supplementation \\
\hline Protein & 50-60 g/Tag, Kostzusammenstellung, Essreihenfolge \\
\hline Eisen & $\begin{array}{l}\text { 50-100 (-600) mg/Tag oral, insbesondere prämenopausale } \\
\text { Frauen }\end{array}$ \\
\hline Vitamin $\mathrm{B}_{12}$ & $350-500 \mu \mathrm{g} /$ Tag oral, gelegentlich 1000-2000 IU s.c./Monat \\
\hline Folsäure & $400 \mu \mathrm{g} /$ Tag oral \\
\hline Calcium & $1000-2000 \mathrm{mg} / \mathrm{Tag}$ oral \\
\hline Vitamin D & 400 IU/Tag oral (<60 Jahre), 700 IU/Tag oral (> 60 Jahre) \\
\hline Vitamin $\mathrm{B}_{1}$ & $100 \mathrm{mg}$ Thiamin/Tag (bei Klinik) \\
\hline Übrige & täglich handelsübliche Multivitaminpräparate \\
\hline Vitamine & \\
\hline
\end{tabular}

sammenstellung ausgeglichen werden. Ein Vitamin- $\mathrm{B}_{1}$-Mangel kann die Ursache für eine Wernicke-Enzephalopathie darstellen. Derartige beobachtete Mangelzustände sind jedoch überwiegend Einzelfälle und eher nach BPD-DS (Duodenalswitch) möglich. Eine entsprechende Substitutionstherapie mit $100 \mathrm{mg}$ Thiamin/Tag, eventuell in Kombination mit Vitamin $\mathrm{B}_{6}$ und $\mathrm{B}_{12}$, über 1-2 Wochen sollte umgehend eingeleitet werden und führt rasch zu einer klinischen Besserung [10].

In der Synopsis der aktuellen Literaturdaten sind Mangelzustände nach RYGB nicht selten und bedürfen einer entsprechenden postoperativen Laboranalytik und Substitution im Nachsorgeprogramm (Tab. 3). Dabei muss beachtet werden, dass bei morbid Adipösen bereits präoperativ Begleitstörungen vorliegen können und mögliche Mangelerscheinungen sich postoperativ nicht linear ausbilden. So können ein Eisenmangel bzw. sHPT durchaus erst 12 Jahre postoperativ manifest werden [21, 23]. Nach gegenwärtigem Kenntnisstand sind im Nachsorgeprogramm malabsorptiver Operationen innerhalb der ersten beiden Jahre vierteljährliche, im 3. und 4. Jahr halbjährliche und dann jährliche bzw. symptombezogene Laborkontrollen empfehlenswert.

\section{Komorbiditätsprognose nach RYGB}

Morbid adipöse Patienten haben nicht nur ein um 29-40\% erhöhtes adjustiertes Risiko, früher zu sterben, sondern weisen zudem mehr als 30 mögliche assoziierte Nebenerkrankungen auf [6, 24, 25]. Viele dieser Störungen können sich nach RYGB bessern oder sogar heilen und sollten durch eine entsprechende internistische Betreuung im Nachsorgeteam begleitet und abgesichert werden. Darunter stellen die Hypertonie-Herzkrankheit, der Typ II Diabetes mellitus (T2 DM), die Hypercholesterinämie und Hyperurikämie, das Schlaf-Apnoe-Syndrom sowie Erkrankungen aus dem psychischen Formenkreis die dominanten Begleiterkrankungen dar, deren Inzidenz nahezu linear mit dem Grad des Übergewichts verbunden ist [26-28].

Buchwald et al. [7] gingen 2004 in einer weiteren Metaanalyse der Frage nach, inwieweit bariatrisch operierte Patienten eine Verbesserung der assoziierten Komorbidität erfahren. Dazu wurden 136 Studien mit insgesamt 22094 Patienten und einem durchschnittlichen Eingangs-BMI von 47 analysiert. Die Autoren fanden 
insgesamt einen EWL von 61\%, mit einer Verbesserung des T2 DM in 77\%, der Hypertonie in 62\%, der Hypercholesterinämie in $71 \%$ und des Schlaf-Apnoe-Syndroms in sogar $86 \%$ der Fälle. Ähnlich wie in dem Swedish Obese Subjects(SOS)-Trial wurden in dieser Analyse jedoch auch in 22-61\% der Fälle weniger effektive bzw. historische (z. B. Vertical Banded Gastroplasty; VBG) Operationsverfahren berücksichtigt.

Schauer et al. [29] fanden nach RYGB mit einem Follow-up von bis zu 30 Monaten eine Verbesserung bzw. Heilung assoziierter Nebenerkrankungen im Hinblick auf eine Hypercholesterinämie bei 33 bzw. $63 \%$, bei GERD bei 24 bzw. 72\%, beim Schlaf-Apnoe-Syndrom bei 19 bzw. 74\%, bei depressiven Erkrankungen bei 47 bzw. $8 \%$ und beim T2 DM sogar bei 18 bzw. $82 \%$ ihrer Patienten.

Insbesondere die Behandlung des T2 DM steht in diesem Zusammenhang im Mittelpunkt des Interesses. MacDonald et al. [30] verglichen 78 morbid adipöse konservativ behandelte Patienten mit 144 RYGB-Operierten und fanden nach einem Follow-up von 6,2 Jahren in der konservativen Gruppe eine Zunahme des T2 DM von 56,4 auf $87,5 \%$ der Patienten. In der operierten Gruppe nahm im Vergleichszeitraum der T2 DM von 31,8\% vor der Operation auf $8,6 \%$ ab, was einem kalkulierten Vorteil von $98 \%$ für die Operierten entspricht. Sugerman et al. [31] fanden 2 Jahre nach RYGB eine Heilungsrate von $83 \%$ für T2 DM, nach 5-7 Jahren von sogar 86\% (Follow-up-Rate 91 und 50\%). Im SOS-Trial [32] wurde eine T2-DM-Heilung nach 10 Jahren für Patienten nach Gastric Banding bei $25,3 \%$, nach VBG in $27,2 \%$ und hochsignifikant häufiger nach RYGB bei $45 \%(\mathrm{p}<0,001)$ beschrieben. Darüber hinaus zeigten operierte Patienten gegenüber der nicht operierten Kontrollgruppe eine deutliche Verbesserung im Hinblick auf eine vorbestehende Triglyzeridämie, Hypertonie-Herzkrankheit und Hyperurikämie $[26,28,33]$.

\section{Bewegungstherapie}

In zwei Studien konnte gezeigt werden, dass Patienten mit einem entsprechenden Bewegungsprogramm nach RYGB eine bessere Gewichtsabnahme verzeichnen und darüber hinaus in validen Erhebungen (z.B. SF-36) eine deutlich verbesserte Lebensqualität angeben $[8,9]$.

Das Trainingsprogramm sollte sowohl kardiovaskuläre als auch bindegewebsrestitutive Gesichtspunkte berücksichtigen. Primär sind gelenk- und wirbelsäulenschonende Übungen angezeigt. Mit zunehmendem Gewichtsverlust kann die muskuloskelettale Entlastung auch die Ausübung von dynamischeren Sportarten erlauben. Vorzugsweise ist der Start des Bewegungsprogramms in der 4. Woche nach RYGB zu empfehlen.

1. Schritt (4.-9. postoperative Woche): Ab der 4. Woche sollten die Patienten neben ihren gewöhnlichen Spaziergängen möglichst 15 min täglich, an 5 Tagen der Woche forciert gehen. 2 Tage pro Woche sollten zunächst der Erholung dienen und werden erst später durch körperliche Aktivität aufgefüllt. Dieser tägliche Ausflug kann um jeweils 2-5 min gesteigert werden. An dieser Stelle empfiehlt sich der Einsatz von Schrittzählern.
Wenn die Patienten vorher schon geschwommen sind, nehmen sie dieses regelmäßige Bewegungsprogramm wieder auf oder beginnen nach den örtlichen Gegebenheiten einmal wöchentlich damit.

2. Schritt (10.-12. postoperative Woche): Zunächst steht noch die kardiovaskuläre Revitalisierung im Vordergrund. Die tägliche Strecke für schnelles Gehen wird schrittweise auf insgesamt 3540 min erweitert. Es genügt, dass nur noch an einem Tag in der Woche pausiert wird.

Die Schwimmstrecke und -dauer kann in dieser Phase langsam gesteigert bzw. verdoppelt werden (ca. $2 \times 45 \mathrm{~min} /$ Woche).

3. Schritt (13.-15. postoperative Woche): Während Gehen und Schwimmen vordergründig kardiovaskulär ausgerichtet sind und den Gewichtsverlust beschleunigen sollen, ist spätestens ab der 12. Woche die Bindegewebsrückbildung zu unterstützen. Es empfiehlt sich, mit einfacher Gymnastik anzufangen. Mitunter können kleine Fußbänke und handelsübliche Gymnastik-Gummibänder hilfreich sein. Der zeitliche Umfang sollte circa 10 min täglich, an 5 Tagen pro Woche betragen. Weiterhin jedoch sollten die Patienten das schnelle Gehen nicht vernachlässigen und zum Ende dieser Phase auf einen Wechsel von etwa 15 min Gymnastik und circa 30 min schnelles Gehen täglich kommen. Viele Selbsthilfegruppen haben in diesem Zusammenhang für ihre Mitglieder Sonderkonditionen in Schwimmhallen und Fitness-Studios vereinbart.

4. Schritt (ab 16. postoperative Woche): Nach der 16. Woche und bei einem planmäßigen Gewichtsverlust können viele Betroffenen mit attraktiven Bewegungsübungen wie Fahrradfahren (Achtung: Standardfahrräder sind bis $100 \mathrm{~kg}$ Traglast, Spezialräder bis $170 \mathrm{~kg}$ Traglast TÜV-geprüft), Nordic Walking und sogar unter Umständen mit Jogging beginnen. Schwimmen bleibt weiterhin ein integraler Bestandteil der Ertüchtigung. Lebenslang sinnvoll ist ein einstudierter Rhythmus von kardiovaskulärer Ertüchtigung und körperlicher Rückbildungsgymnastik.

\section{Schlussfolgerung}

Zusammenfassend lässt sich feststellen, dass der Erfolg nach RYGB wegen morbider Adipositas im Hinblick auf Gewichtsverlust, Verbesserung der Adipositas-assoziierten Komorbidität und psychosoziale Integration entscheidend von einem strukturierten Nachsorgeprogramm abhängt. Aufgrund der malabsorptiven Komponente des Verfahrens können Patienten nach RYGB nutritive Mangelzustände erleiden, die eine sichere Supplementation und strukturierte Laboranalytik im Follow-up erfordern. Es konnte gezeigt werden, dass Gewichtsverlust und Komorbiditätsverbesserung nach RYGB streng mit einem physikalischen Trainingsprogramm korrelieren, obgleich auch hier die Literaturdatenlage verbessert werden muss.

\section{Disclosure}

Die Autoren geben keine Interessenkonflikte an 


\section{Literatur}

1 Ogden CL, Caroll MD, Curtin LR, McDowell MA, Tabak CJ, Flegel KM: Prevalence of overweight and obesity in the United States, 19992004. JAMA 2006;295:1549-1555.

$\checkmark 2$ Buchwald H, Williams SE: Bariatric surgery worldwide 2003. Obes Surg 2004;14:1157-1164.

3 Santry HP, Gillen DL, Lauderdale DS: Trends in bariatric surgical procedures. JAMA 2005;294: 1909-1917.

4 Flum DR, Salem L, Elrod JA, Dellinger EP, Cheadle A, Chan L: Early mortality among medicare beneficiaries undergoing bariatric surgical procedures. JAMA 2005;294:1903-1908.

5 Buchwald H, Estok R, Fahrbach K, Banel D, Sledge I: Trends in mortality in bariatric surgery: a systemic review and meta-analysis. Surgery 2007;142:621-632.

6 Sjöström L, Narbro K, Sjöström CD, Karason K, Larsson B, Wedel H, Lystig T, Sullivan M, Bouchard C, Carlsson B, Bengtsson C, et al, and the SOS-Study: Effects of bariatric surgery on mortality in Swedish obese subjects. N Engl J Med 2007;357:741-752

7 Buchwald H, Avidor Y, Braunwald E, Jensen MD, Pories W, Fahrbach K, Schoelles K: Bariatric surgery: a systematic review and meta-analysis. JAMA 2004;292:1724-1737.

8 Welch G, Wesolowski C, Piepul B, Kuhn J, Romanelli J, Garb J: Physical activity predicts weight loss following gastric bypass surgery; findings from a support survey. Obes Surg 2008;18:517-524.

9 Bond DS, Phelan S, Wolfe LG, Evans RK, Meador JG, Kellum JM, Maher JW, Wing RR: Becoming physically active after bariatric surgery is associated with improved weight loss and healthrelated quality of life. Obesity 2008;DOI:10.1038/ oby.2008.501

-10 Ludwig K, Bernhardt J: Potentielle Mangelerscheinungen und Supplementation nach Magen-Bypass. Chir Gastroenterol 2007;23(suppl 1):30-34

11 Brolin RE, Leung M: Survey of vitamin and mineral supplementation after gastric bypass and biliopancreatic diversion for morbid obesity. Obes Surg 1999;9:150-154.

12 Mallory GN, Macgregor AM, Rand CS: The influence of dumping on weight loss after gastric restrictive surgery for morbid obesity. Obes Surg 1996;6:474-478.

13 Weiner RA, Pomhoff I, Schramm M, Matic S: Complications after laparoscopic Roux-en-Y gastric bypass. Chir Gastroenterol 2005;21(suppl 1): 13-22.

14 Choban PS, Flancebaum L: The effect of Roux limb lengths on outcome after Roux-en-Y gastric bypass: a prospective, randomized clinical trial. Obes Surg 2002;12:540-545.

15 Brolin RE, Kenler HA, Gorman JH, Cody RP: Long-limb gastric bypass in the superobese. Ann Surg 1992;215:387-395.

6 Bloomberg RD, Fleishman A, Nalle JE, Herron DM, Kini S: Nutritional deficiencies following bariatric surgery: what have we learned? Obes Surg 2005;15:145-154.

17 Skroubis G, Sakellaropoulos G, Pouggouras K, Med N, Nikiforidis G, Kalfarentzos F: Comparison of nutritional deficiencies after Roux-en-Y gastric bypass and after biliopancreatic diversion. Obes Surg 2002;12:551-558.

18 Brolin RE, Gorman JH, Gorman RC, Petschenik AJ, Bradly LJ, Kenler HA, Cody RP: Are vita$\min B_{12}$ and Folate deficiency clinically important after roux-en-Y gastric bypass? J Gastrointest Surg 1998;2:436-442.

19 Brolin RE, LaMarca LB, Kenler HA, Cody RP: Malabsorptive gastric bypass in patients with superobesity. J Gastrointest Surg 2002;6:195-203.

20. Goode LR, Brolin RE, Chowdhury HA, Shapses SA: Bone and gastric bypass surgery: effects of dietary calcium and vitamin D. Obes Res 2004;12:40-47.

21 Johnson JM, Maher JW, DeMaria EJ, Downs RW, Wolfe LG, Kellum JM: The long-term effects of gastric bypass on vitamin D metabolism. Ann Surg 2006;243:701-705.

22 Coates PS, Fernstrom JD, Fernstrom MH, Schauer PR, Greenspan SL: Gastric bypass surgery for morbid obesity leads to increase in bone turnover and decrease in bone mass. J Clin Endocrinol Metab 2004;89:1061-1065.

23 DiGiorgi M, Daud A, Inabnet WB, Schrope B, Urban-Skuro M, Restuccia N, Bessler M: Markers of bone and calcium metabolism following gastric bypass and laparoscopic adjustable gastric banding. Obes Surg 2008;18:1144-1148.
Adams TD, Gress RE, Smith SC, Halverson RC Simper C, Rosamond WD, LaMonte MJ, Stroup AM, Hunt SC: Long-term mortality after gastric bypass surgery. N Engl J Med 2007;357:753-761.

25 Christou NV, Sampalis JS, Liberman M, Look D, Auger S, McLean APH, MacLean LD: Surgery decreases long-term mortality, morbidity, and health care use in morbidly obese patients. Ann Surg 2004; 240:416-424.

26 Peluso L, Vanek VW: Efficacy of gastric bypass in the treatment of obesity-related comorbidities. Nutr Clin Pract 2007;22:22-28.

27 Maggard MA, Shugarman LR, Suttorp M, Maglione M, Sugerman HJ, Livingston EH, Nguyen NT, Li Z, Mojica WA, Hilton L, Rhodes S, Morton SC, Shekelle PG: Meta-analysis: surgical treatment of obesity. Ann Intern Med 2005;142: 547-559.

28 Mokdad AH, Ford ES, Bowman BA, Dietz WH, Vinicior F, Bales VS, Marks JS: Prevalence of obesity, diabetes, and obesity-related health risk factors. JAMA 2003;289:76-79.

29 Schauer PR, Ikramudin S, Gourash W, Ramanathan R, Luketich J: Outcomes after laparoscopic Roux-en-Y gastric bypass for morbid obesity. Ann Surg 2000;232:515-529.

30 MacDonald KG Jr, Long SD, Swanson MS, Brown BM, Morris P, Dohm GL, Pories WJ: The gastric bypass operation reduces the progression and mortality of non-insulin-dependent diabetes mellitus. J Gastrointest Surg 1997;3:213-220.

31 Sugerman HJ, Wolfe LG, Sica DA, Clore JN: Diabetes and hypertension in severe obesity and effects of gastric bypass-induced weight loss. Ann Surg 2003;237:751-758.

32 Sjöström L, Lindroos AK, Peltonen M, Torgerson J, Bouchard C, Carlsson B, Dahlgren S, Larsson B, Narbro K, Sjöström CD, Sullivan M, Wedel H: Lifestyle, diabetes, and cardiovascular risk factors 10 years after bariatric surgery. N Engl J Med 2004;351:2683-2693.

33 Bender R, Jöckel KH, Richter B, Spraul M, Berger M: Body weight, blood pressure, and mortality in a cohort of obese patients. Am J Epidemiol 2002;156:239-245. 